\title{
Gauge symmetry and the generalization of Hirota's bilinear method
}

\author{
Jarmo HIETARINTA \\ Department of Physics \\ FIN-20014 Turku University, FINLAND \\ e-mail: hietarin@utu.fi
}

\begin{abstract}
One of the most powerful methods for finding and solving integrable nonlinear partial differential equations is Hirota's bilinear method. The idea behind it is to make first a nonlinear change in the dependent variables after which multisoliton solutions of integrable systems can be expressed as polynomials of exponentials $e^{\eta_{i}}$ where the $\eta_{i}^{\prime} s$ are linear in the independent variables. Among all quadratic expressions homogeneous in the derivatives, Hirota's bilinear form can be isolated by a gauge symmetry: it is the only one that is invariant under $f_{\alpha} \rightarrow e^{\theta} f_{\alpha}$ where $\theta$ is linear in the variables. This suggest a generalization to multilinear equations using the same gauge symmetry. The set of gauge invariant multilinear differential equations can then be studied and integrable equations identified e.g. by the Painlevé method. Some interesting new equations have been found in this way.
\end{abstract}

\section{Introduction}

Since the late 1960's solitons have been studied intensively, and the related theory has by now been firmly established. Solitons (which by definition are localized and stable travelling-wave solutions, which interact elastically) have also been seen experimentally, from kilometers wide internal waves in oceans to very short pulses in optical fibers.

Solitons appear only in integrable nonlinear PDE's and since the conditions for integrability are rather demanding, integrable systems are rare (which in no way limits their influence [1]). One important problem is, therefore, the search for new integrable systems. This can be done in many ways: take any property that is common to integrable systems and try to generalize it while keeping the good implications. Some of the useful properties of soliton systems are:

- There are infinite number of conserved quantities.

- The nonlinear evolution equation arises from a consistence condition of two linear equations (Lax pair).

- One can construct closed form multisoliton solutions.

- One can solve the initial value problem (inverse scattering transform).

There seems to be some kind of symmetry property behind each of these properties. In this talk we would like to point out that there is also a gauge symmetry behind the multisoliton property, this becomes clear when one uses Hirota's bilinear method in constructing the solutions. Futhermore, it is possible to make a natural generalization of Hirota's method while keeping this symmetry [2].

Copyright (c) 1996 by Mathematical Ukraina Publisher. All rights of reproduction in any form reserved. 
In the next section we will give a short description of Hirota's bilinear method and then introduce the gauge symmetry. Next the symmetry is generalized to multilinear systems. A class of such multilinear systems has been searched for integrable equations, using the Painlevé method, and we recall some results here [3].

\section{Hirota's bilinear method}

We will first briefly discuss Hirota's very effective method of constructing multisoliton solutions to integrable equations (for further details, see e.g. [4]).

The first step is a transformation to Hirota's "bilinear form". As an example let us consider the Korteweg - de Vries (KdV) equation

$$
u_{x x x}+6 u u_{x}+u_{t}=0 .
$$

Let us introduce the dependent variable transformation

$$
u=2 \partial_{x}^{2} \log F,
$$

and then one can write (1) in the following bilinear form (after one integration):

$$
F_{x x x x} F-4 F_{x x x} F_{x}+3 F_{x x}^{2}+F_{x t} F-F_{x} F_{t}=0 .
$$

This does not look simpler than (1) but one can write it in a condensed form using the Hirota $D$ operator:

$$
\left(D_{x}^{4}+D_{x} D_{t}\right) F \cdot F=0
$$

here $D_{x} \equiv \partial_{x_{1}}-\partial_{x_{2}}$ acts on a pair of functions (the 'dot product') antisymmetrically:

$$
D_{x}^{n} f \cdot g=\left.\left(\partial_{x_{1}}-\partial_{x_{2}}\right)^{n} f\left(x_{1}\right) g\left(x_{2}\right)\right|_{x_{2}=x_{1}=x} .
$$

The minus sign here is crucial.

Clearly the bilinear form of the $\mathrm{KdV}$-equation belongs to the class

$$
P\left(D_{x}, D_{y}, \ldots\right) F \cdot F=0, \quad P(0)=0 .
$$

For this class of equations multisoliton solutions are indeed simple in terms of $F$, as opposed to $u$. It is easy to see, that due to the antisymmetry in (5) $F_{1}=1+e^{\eta}(\eta=p \cdot x)$ is a solution of (6), if just the parameters $p$ satisfy a dispersion relation $P(p)=0 . F_{1}$ is the one-soliton solution (1SS) and substitution to (2) yields the standard result for $u$.

The two-soliton solution (2SS) is a kind of linear superposition of two 1SS's $\left(\eta_{i}=p_{i} \cdot x\right)$ :

$$
F_{2}=1+e^{\eta_{1}}+e^{\eta_{2}}+A_{12} e^{\eta_{1}+\eta_{2}} .
$$

The "phase factor" $A_{12}$ is given by $-P\left(p_{1}-p_{2}\right) / P\left(p_{1}+p_{2}\right)$.

The above works for any polynomial $P$. However, a three soliton solution can be constructed only for integrable equations. The only natural 3SS generalization, having the correct 2SS limits, is

$$
\begin{aligned}
F= & 1+e^{\eta_{1}}+e^{\eta_{2}}+e^{\eta_{3}}+ \\
& A_{12} e^{\eta_{1}+\eta_{2}}+A_{13} e^{\eta_{1}+\eta_{3}}+A_{23} e^{\eta_{2}+\eta_{3}}+A_{12} A_{13} A_{23} e^{\eta_{1}+\eta_{2}+\eta_{3}} .
\end{aligned}
$$


However, this is a solution of (6) only if certain functional relations involving $P$ are satisfied. This provides a method for searching for new candidates for integrability: find those $P$ 's that solve the resulting equations. This method has turned out to be quite efficient, especially since the form of $P$ is not restricted in any way at the beginning. The same idea works for some other types of bilinear equations as well (modified KdV, sine-Gordon, nonlinear Schrödinger). [For some results, see [5].]

\section{Gauge invariance in Hirota's method}

\subsection{Bilinear}

First notice that the relation of the 'physical' variable $u$ to the Hirota's function $F$ given in (2) is such that $u$ is invariant under $F \rightarrow e^{q \cdot x} F$. This gauge invariance is also evident in the soliton solutions, an N-soliton solution is form invariant under $F \rightarrow F \exp \left(-\sum_{i} p_{i} \cdot x\right)$.

In fact, one can define Hirota's bilinear form through the requirement of gauge invariance: if we take any expression quadratic in the dependent variables and homogeneous in the derivatives

$$
A_{n}(f, g):=\sum_{i=0}^{n} c_{i}\left(\partial_{x}^{i} f\right)\left(\partial_{x}^{n-i} g\right),
$$

then it turns out that $A_{n}$ is gauge invariant, i.e.,

$$
A_{n}\left(e^{\theta} f, e^{\theta} g\right)=e^{2 \theta} A_{n}(f, g), \text { where } \theta=q x,
$$

if and only if

$$
c_{i}=(-1)^{i}\left(\begin{array}{c}
N \\
i
\end{array}\right) c_{0},
$$

so that in the end $A_{n}$ can be written as

$$
A_{n}(f, g)=c_{0} D_{x}^{n} f \cdot g .
$$

\subsection{From bilinear to multilinear}

The above observation can be used as a guideline in generalizing Hirota's method: let us define multilinear operators also by a gauge condition.

For an expression cubic in dependent variables and homogeneous (of degree $N$ ) in the $x$-derivatives

$$
B_{N}(f, g, h):=\sum_{k+l+m=N} c_{k l m}\left(\partial_{x}^{k} f\right)\left(\partial_{x}^{l} g\right)\left(\partial_{x}^{m} h\right),
$$

the gauge invariance requirement is

$$
\sum_{k+l+m=N} c_{k l m}\left(\partial_{x}^{k} e^{\eta} f\right)\left(\partial_{x}^{l} e^{\eta} g\right)\left(\partial_{x}^{m} e^{\eta} h\right)=e^{3 \eta} \sum_{k+l+m=N} c_{k l m}\left(\partial_{x}^{k} f\right)\left(\partial_{x}^{l} g\right)\left(\partial_{x}^{m} h\right) .
$$

where $\eta=x p+\eta_{0}$. This again introduces conditions on $c_{k l m}$ and one finds [2] that a basis for such gauge invariant operators can given by the $N+1$ combinations: $D_{12}^{n} D_{13}^{N-n}$ for $n=0, \ldots, N$ where, for example,

$$
D_{12} f \cdot g \cdot h=\left.\left(\partial_{x_{1}}-\partial_{x_{2}}\right) f\left(x_{1}\right) g\left(x_{2}\right) h\left(x_{3}\right)\right|_{x_{i}=x} .
$$


A more symmetric basis is given by $T^{n}\left(T^{*}\right)^{N-n}$, where

$$
T=\partial_{1}+j \partial_{2}+j^{2} \partial_{3}, \quad T^{*}=\partial_{1}+j^{2} \partial_{2}+j \partial_{3}, \quad j=e^{2 i \pi / 3} .
$$

The above generalizes to any order of multilinearity by introducing the set of $n(n-1) / 2$ operators $D_{i j}\left(n-1\right.$ of them independent) acting on $n$-tuple dot-products $D_{i j} f_{1} \cdot f_{2} \cdot \ldots \cdot f_{n}$, or the 'symmetric' operators:

$$
M_{n}^{m}=\sum_{k=0}^{n-1} e^{2 \pi i k m / n} \partial_{k+1}, \text { for } 0<m<n .
$$

For example, $D=M_{2}^{1}, T=M_{3}^{1}, T^{*}=M_{3}^{2}$.

\section{Searching for integrable trilinear equations}

Once a class of interesting equations has been defined, one can search for integrable equation within that class. For multilinear equations his can be done either by asking for multisoliton solution or by Painlevé analysis. The latter was done for trilinear equations of type $P\left(T, T^{*}\right) F \cdot F \cdot F=0$ in $[2,3]$, here we will just mention some of the most interesting equations found in that work.

The first new equation passing the Painlevé test was found at degree 5 in the $T$ 's:

$$
\left(T_{x}^{4} T_{y}^{*}+8 T_{x}^{3} T_{y} T_{x}^{*}+9 T_{y}^{3}+9 T_{x}^{2} T_{z}\right) F \cdot F \cdot F=0 .
$$

Putting $F=e^{g}$ we obtain its nonlinear version

$$
g_{x x x x y}+8 g_{x x y} g_{x x}+4 g_{x y} g_{x x x}+g_{y y y}+g_{x x z}=0 .
$$

This carries some resemblance with the KP equation. In this case nontrivial three soliton solutions have also been shown to exist.

The fifth-order equation in the Lax hierarchy was found at degree 6 :

$$
\left(20 T_{x}^{3} T_{x}^{* 3}+7 T_{x}^{6}+\alpha T_{x}^{2} T_{x}^{* 2}+27 T_{x}^{*} T_{y}\right) F \cdot F \cdot F=0,
$$

or in nonlinear form $\left(u=2 \partial_{x}^{2} \log F\right.$, derivate once):

$$
u_{5 x}+10 u u_{x x x}+20 u_{x} u_{x x}+30 u^{2} u_{x}+27 \alpha\left(u_{x x x}+6 u u_{x}\right)+u_{y}=0 .
$$

Thus the Lax-5 equation does not have a simple bilinear form like $\mathrm{KdV}$ but a trilinear one.

New equations passing the Painlevé test were found also at degrees 8 and 10:

$$
\left(4 T_{x}^{7} T_{x}^{*}+5 T_{x}^{4} T_{x}^{* 4}+\alpha\left(20 T_{x}^{3} T_{x}^{* 3}+7 T_{x}^{6}\right)+9 \beta T_{x}^{2} T_{x}^{*} T_{y}^{*}+\frac{9}{2} \alpha \beta T_{x} T_{y}^{*}\right) F \cdot F \cdot F=0,
$$

whose nonlinear form is $\left(u=6 \partial_{x} \log F\right)$

$$
\begin{aligned}
& u_{7 x}+6 u_{5 x} u_{x}+10 u_{4 x} u_{x x}+5 u_{x x x}^{2}+10 u_{x x x} u_{x}^{2}+10 u_{x x}^{2} u_{x}+\frac{5}{3} u_{x}^{4}+ \\
& \alpha\left(3 u_{5 x}+10 u_{x x x} u_{x}+5 u_{x x}^{2}+\frac{10}{3} u_{x}^{3}\right)+\beta\left(u_{x x y}+u_{x} u_{y}\right)+\frac{1}{2} \alpha \beta u_{y}=0,
\end{aligned}
$$

and

$$
\begin{aligned}
\left(5 T_{x}^{8} T_{x}^{* 2}+4 T_{x}^{5} T_{x}^{* 5}+\alpha\left(4 T_{x}^{7} T_{x}^{*}+5 T_{x}^{4} T_{x}^{* 4}\right)+\beta\left(20 T_{x}^{3} T_{x}^{* 2} T_{y}^{*}+7 T_{x}^{5} T_{y}\right)+\right. & \\
\left.6 \alpha \beta T_{x}^{2} T_{x}^{*} T_{y}^{*}+\frac{3}{2} \beta^{2} T_{y} T_{y}^{*}\right) F \cdot F \cdot F & =0
\end{aligned}
$$


with the nonlinear form $(g=6 \log F)$

$$
\begin{aligned}
& g_{10 x}+10 g_{8 x} g_{2 x}+20 g_{7 x} g_{3 x}+30 g_{6 x}\left(g_{4 x}+g_{2 x}^{2}\right)+25 g_{5 x}\left(g_{5 x}+4 g_{3 x} g_{2 x}\right)+ \\
& 50 g_{4 x}^{2} g_{2 x}+50 g_{4 x} g_{3 x}^{2}+\frac{100}{3} g_{4 x} g_{2 x}^{3}+50 g_{3 x}^{2} g_{2 x}^{2}+\frac{10}{3} g_{2 x}^{5}+ \\
& \alpha\left(g_{8 x}+6 g_{6 x} g_{2 x}+10 g_{5 x} g_{3 x}+5 g_{4 x}^{2}+10 g_{4 x} g_{2 x}^{2}+10 g_{3 x}^{2} g_{2 x}+\frac{5}{3} g_{2 x}^{4}\right)+ \\
& \beta\left(3 g_{5 x y}+5 g_{3 x} g_{x x y}+\frac{10}{3}\left(g_{4 x} g_{x y}+g_{x x}^{2} g_{x y}+2 g_{x x} g_{x x x y}\right)\right)+ \\
& \quad \frac{2}{3} \alpha \beta\left(g_{x x x y}+g_{x x} g_{x y}\right)+\frac{1}{6} \beta^{2} g_{y y}=0 .
\end{aligned}
$$

\section{Equations with a missing leading term}

The nonlinear equation corresponding to (6) is obtained by the substitution $F=e^{g}$ and leads to

$$
P(D) e^{g} \cdot e^{g}=e^{2 g}[(P(\partial)+P(-\partial)) g+\text { nonlinear terms }] .
$$

Since the odd parts of $P$ do not contribute (even for the nonlinear terms) we see that in the bilinear case the leading (linear) part is given by the polynomial $P$ itself, which also defines the dispersion relation, as was shown before.

With trilinear operators the situation is more complicated. The observation above, that only the even part of $P$ contributes in the bilinear case, is now replaced by the following

$$
T^{n} T^{* m} F \cdot F \cdot F=0, \text { unless } n+2 m=0 \quad(\bmod 3) .
$$

For the leading part of the nonlinear equation corresponding to a trilinear equation this implies that if $n+2 m=0 \quad(\bmod 3)$

$$
T^{n} T^{* m} e^{g} \cdot e^{g} \cdot e^{g}=3 e^{3 g}\left[\partial^{n+m}+\text { nonlinear terms }\right] .
$$

Thus the leading part is only sensitive to $n+m$, but the nonlinear part depends on both $n$ and $m$.

The gives to the novel possibility that the leading term may now vanish even when the nonlinear terms do not. The simplest one dimensional example of this is obtained at degree 6 when $n=6, m=0$ and $n=3, m=3$ both satisfy the condition $n+2 m=0$ $(\bmod 3)$. Thus the equation

$$
\left(T_{x}^{6}-T_{x}^{3} T_{x}^{* 3}\right) F \cdot F \cdot F=0,
$$

which in determinant form reads [6]

$$
\left|\begin{array}{ccc}
F^{\prime \prime \prime \prime} & F^{\prime \prime \prime} & F^{\prime \prime} \\
F^{\prime \prime \prime} & F^{\prime \prime} & F^{\prime} \\
F^{\prime \prime} & F^{\prime} & F
\end{array}\right|=0 .
$$

is of degree six, but there is no sixth order leading term. Another examples of this type is given by the second order PDE

$$
\left(T_{x} T_{y}^{*}-T_{y} T_{x}^{*}\right) F \cdot G \cdot H=0,
$$


or in determinant form

$$
\left|\begin{array}{lll}
F & F_{x} & F_{y} \\
G & G_{x} & G_{y} \\
H & H_{x} & H_{y}
\end{array}\right|=0 .
$$

which only contains first order terms.

The Monge-Ampère equation $w_{x y}^{2}-w_{x x} w_{y y}=0$ belongs also to this class, it is fourth order but has only second order derivatives. It can be written as (with $F=e^{w}$ )

$$
\left(T_{x} T_{x}^{*} T_{y} T_{y}^{*}-T_{x}^{2} T_{y}^{* 2}\right) F \cdot F \cdot F=0
$$

or in determinant form,

$$
\left|\begin{array}{ccc}
F_{y y} & F_{y} & F_{x y} \\
F_{y} & F & F_{x} \\
F_{x y} & F_{x} & F_{x x}
\end{array}\right|=0 .
$$

\section{Conclusion}

We have here discussed an extension of Hirota's bilinear formalism leading to any degree of multilinearity. The main guideline in this generalization has been gauge-invariance: the original nonlinear equation should be transformed into a form that is invariant under a gauge transformation $f_{i} \rightarrow e^{a \cdot x} f_{i}$.

The only new class of equations that has been studied in detail is $P\left(T, T^{*}\right) F \cdot F \cdot F=0$. A search of integrable equations in this class (and with nonvanishing leading term) has been made using the Painlevé test and new integrable equations have been discovered.

Many things that have been done for bilinear equations still remain to be done for trilinear (and higher multilinear) ones, for example classification of integrable multicomponent equations (i.e. those that contain several dependent variables $F, G, \ldots$ ), and the study of multisoliton solutions.

The interesting new class of equations with missing leading term is still completely unexplored territory from the multilinear point of view. Another interesting direction is to apply multilinear techniques to difference equations.

\section{References}

[1] Calogero F., Why are certain nonlinear PDE's both widely applicable and integrable? in: What is integrability? (V.E. Zakharov,ed), Springer, 1991, 1-62.

[2] Grammaticos B., Ramani A. and Hietarinta J., Phys. Lett. A, 1994, V.190, 65-70.

[3] Hietarinta J., Grammaticos B. and Ramani A., Integrable Trilinear PDE's, in: Nonlinear Evolution Equations \& Dynamical Systems, NEEDS '94', eds. V.G. Makhankov, A.R. Bishop and D.D. Holm, (World Scientific, 1995), 54-63.

[4] Hietarinta J., Hirota's bilinear method and partial integrability, in: Partially Integrable Equations in Physics, eds. R. Conte and N. Boccara, (Kluwer, 1990), 459-478.

[5] Hietarinta J., J. Math. Phys., 1987, V.28, 1732, 2094, 2586; ibid 1988, V.29, 628.

[6] Matsukidaira J., Satsuma J., Strampp W., Phys. Lett. A, 1990, V.147, 467. 